\title{
Surgical Reconstruction in Chronic Posterior Cruciate Ligament Injuries of the Knee
}

\author{
Che Ahmad Aa , Najmi NMFa, Samsudin OC ${ }^{b}$, Yeap JKc, Hyzan MYd \\ aDepartment of Orthopaedics, Traumatology \& Rehabilitation, Kulliyyah of Medicine, International Islamic \\ University Malaysia, Kuantan, Pahang \\ bDepartment of Orthopaedics \& Traumatology, Faculty of Medicine, Hospital University Kebangsaan Malaysia, \\ Kuala Lumpur \\ 'Tawakkal Hospital, Kuala Lumpur \\ dSunway Medical Centre, Kuala Lumpur
}

\begin{abstract}
Introduction: This is a cross-sectional study to evaluate the outcome of posterior cruciate ligament reconstruction in chronic injuries using ipsilateral Bone-patella tendon-Bone graft in nine patients, between January 2000 and January 2003 at our institution. Methods: Eight male and one female patient; their ages ranged from 18 to 36 years old. The surgery was done at an average of 17.2 months (range from 3 and 40 months) post injury. All patients were followed up for a minimum of two years period. The assessment was done at twelve and twenty-four months using Lysholm knee score, IKDC knee examination evaluation score and arthrometric measurement with KT-2000. Results: The result showed only moderate success in restoration of ligament stability with $73.4 \%$ satisfactory objective results. However, the functional improvement and patient satisfaction is only $55 \%$. The incidences of degenerative changes were $89 \%$ at the time of operation. Conclusion: Surgical reconstruction of chronic Posterior Cruciate Ligament restored satisfactory stability of the knee but the functional outcome was only moderate.
\end{abstract}

KEYWORDS: Posterior cruciate ligament, surgical reconstruction, chronic ligament injury, knee instability

\section{INTRODUCTION}

Injury of posterior cruciate ligament (PCL) of the knee is relatively uncommon compared with anterior cruciate ligament. The basic science studies of the biomechanics of the posterior cruciate ligament are also limited. Literature on posterior cruciate ligament tear are less abundant and more difficult to interpret. In addition, the posterior cruciate ligament poses difficult surgical reconstruction because of its complex structure and relative inaccessibility. There has been a report of ruptured popliteal artery pseudoaneurysm following PCL reconstruction. ${ }^{1}$

The treatment of posterior cruciate ligament injury remains controversial. The results of posterior cruciate ligament reconstruction in the literature vary. Posterior cruciate ligament has been shown to have a better healing potential compared to anterior cruicate

Corresponding author:

Assoc. Prof. Dr. Aminudin Che Ahmad

Department of Orthopaedics, Traumatology \&

Rehabilitation

Kulliyyah of Medicine

International Islamic University Malaysia

Bandar Indera Mahkota

25200 KUANTAN, Pahang, Malaysia

Tel: $+609-5706137$

Fax: +609-51444451

Email: caamin@gmail.com ligament. $^{2}$ Surgical reconstruction is usually recommended only in complete PCL tear with pain or instability and combined ligament injuries. ${ }^{3,4}$ With improved arthroscopic instruments and better surgical technique, arthroscopic reconstruction has evolved as the method of choice for the treatment of PCL tear.

The question is whether surgical reconstruction of posterior cruciate ligament gives better results than non-operative treatment in chronic injuries. This study retrospectively evaluates the clinical outcome of knees with chronic posterior cruciate ligament laxity in 9 patients who had arthroscopic single bundle reconstruction using ipsilateral bone-tendon-patella graft at our institution with at least two years followup.

\section{MATERIALS AND METHODS}

From January 2000 to January 2003, a total of 9 patients underwent posterior cruciate ligament reconstruction of the knee using ipsilateral bonepatella tendon-bone graft at Hospital Universiti Kebangsaan Malaysia, Kuala Lumpur. Patients have a mean age of 27 years old (range 18 to 33 years). The most common causes of the injuries were road traffic accident and sport related injury. The patients' age, sex, occupation and mechanism of injury were noted.

Inclusion criteria for this study include age over 18 years old, no previous surgery and using ipsilateral patella tendon bone graft. Consent was obtained in all patients to be included in this study. All patients 
presented with pain and instability of the affected knee. Plain anterior-posterior (AP) and lateral radiographs were performed to exclude avulsion injuries of posterior cruciate ligament or any other associated intra-articular fractures. Three of the patients had magnetic resonance (MR) imaging evaluation to look for associated injuries. Three other patients had arthroscopic evaluation prior to reconstruction.

Acute injury is defined as that occuring within 6 weeks and chronic injury is defined as that occuring after 6 weeks. None of the cases was done in the acute period. Surgery was performed at an average of 17.3 months after injury, with a range of 3 months to 40 months.

\section{Surgical Techniques}

The posterior cruciate ligament reconstruction procedure described is done using ipsilateral bonepatella tendon bone autogenous graft by arthroscopic assisted technique. The patient is under either combined spinal epidural (CSE) or epidural anaesthesia and in a supine position. A high tourniquet is used. The affected leg is laid over the end of the table with a sandbag below the thigh to help maintain $90^{\circ}$ to $100^{\circ}$ of knee flexion during procedure. The normal leg is put on lithotomy position to allow ample working space. The areas cleaned include the knee, extending proximal to the upper thigh and distally to the tips of the toe. Standard draping is done as for any knee surgery. The leg and the foot are wrapped in sterile sheets and left free. Systematic arthroscopic examination of the knee performed using standard anterolateral and anteromedial portals and the findings are noted. The remnant of the posterior cruciate ligament stump are removed, soft tissue debrided and any meniscus tear is repaired or trimmed.

\section{Graft Harvest}

To harvest the central-third patella tendon, a longitudinal midline, skin incision is made on the anterior aspect of the knee. Starting at the inferior tip of the patella and extending distally over the tibial tuberosity. The deep fascia is incised in the line of skin incision and the subcutaneous fat was dissected to expose the patella tendon, lower patella and the tibial tuberosity. The graft is then harvested with a 10 millimeter wide central portion of the patella tendon, a block of bone each from the patella $(20 \mathrm{~mm})$ and the tibia $(25 \mathrm{~mm})$. The graft is then shaped and contoured to the appropriate size (10 or 11 millimeter trial). The bone plug which is to be secured in the femoral tunnel is shortened to approximately 18 $\mathrm{mm}$ with bullet shape to make intra-articular passage easier. Two holes drilled in each bone plug and tagged loosely with Dexon 1 sutures.

\section{Tibial Tunnel Preparation}

For making the tibial tunnel, the Arthrex ${ }^{\circledR}$ drill guide system is used. The guide is inserted through the anteromedial portal and passed through the notch. The guide tip is placed 10 to 12 millimeter below the joint line in the posterior cruciate ligament facet. Then the drill is oriented approximately 45$60^{\circ}$ to the articular surface of the tibia, entering just inferior and medial to the tibial tuberosity. A more perpendicular angle will create too much acute angle at the posterior tibia that may cause abrasion to the graft. The guide pin is adjusted so that it will protrude from the tip of the drill 10 millimeter less than the distance measured on the guide system helps prevent over drilling. The pin is tapped in the last $1 \mathrm{~cm}$ to help prevent penetration. A posteromedial portal is created to place a shaver to protect the neurovascular structures from pin penetration during advancement and reaming. The posteromedial portal also helps in tissue clearance at the posterior tibia. The guide pin should exit at the junction of the middle and distal one third of the posterior tibial sulcus slightly lateral to the posterior cruciate ligament insertion. A proper placement of the guide wire can be confirmed with help of image intensifier. Through the anteromedial portal, a tibial drill guide is positioned approximately $1.5 \mathrm{~cm}$ below the posterior cruciate ligament insertion. The tibial tunnel is reamed, according to the graft size, with the knee in 90 degree flexion.

\section{Femoral Tunnel Preparation}

The femoral tunnel placement is technically less difficult than the tibial side but more critical to procedures' success. The femoral guide pin entrance pin requires $3 \mathrm{~cm}$ longitudinal incision midway between the patella and medial femoral epicondyle. The vastus medialis is elevated superiorly and subperiosteal window is developed over the metaphyseal flare. The guide pin is initiated $1.5 \mathrm{~cm}$ from the articular surface. The exit point i.e. the femoral anatomical attachment in the intercondylar notch should be approximately $10 \mathrm{~mm}$ posterior to articular junction at the 1 o'clock position on the right knee and 11 o'clock position on the left knee.

Isometric point for the posterior cruciate ligament is not as well defined as those for anterior cruciate ligament. Many authors agree that this point reproduces the centre of large anterior and central portions of the posterior cruciate ligament, more anterior posterior orientation and tighten its flexion. An appropriate size $(9 \mathrm{~mm}$ or $10 \mathrm{~mm})$ femoral tunnel corresponding to the size of the graft is reamed and the edges are rasped.

\section{Graft Passage and Fixation}

Under arthroscopic visualization, the suture is pulled and the attached graft passes across the joint into femoral tunnel until the insertion mark disappears into the tunnel. The femoral attachment is fixed first with a cannulated interference screw. Then pretensioning of the graft is performed by flexing and extending the knee several cycles, from zero to full 
flexion. The tibial plug is fixed while pulling the graft and fixed at $50-70^{\circ}$ flexions.

\section{Associated Injury Reconstructions}

Posterolateral complex reconstruction performed using Clancy $^{5}$ technique of bicep femoris tenodesis. Meniscus is repaired using outside-in technique.

\section{Closure}

The drain was inserted into the knee joint. The subcutaneous tissue closed with absorbable suture and skin is closed by 3/0 Dafilon suture. Lastly the joint infiltrated with $20 \mathrm{ml} 0.5 \%$ Marcaine for pain control. Cotton dressing and crepe bandage is applied over the wound.

\section{Rehabilitation}

All patients in this study underwent rehabilitation following a protocol for posterior cruciate ligament reconstruction popularized by Paulos ${ }^{6}$ and supervised by a dedicated physiotherapist.

\section{Follow up}

All the patients were followed up from twelve to twenty-four months after operation and had already gone through scheduled rehabilitation protocol. Patients were assessed subjectively by using Lysholm knee score and IKDC 2000 grading at twelve and twenty-four months, and objectively by using KT2000 arthrometric measurement also after twelve and twenty-four months.

The Lysholm knee score is designed to evaluate symptoms (limp, support, locking, instability, pain, swelling, star climbing and squatting). They are further graded excellent with a score at 95-100, good (84-94), fair (65-83) and poor is less than 65.7 In International Knee Documentation Committee (IKDC 2000) knee evaluation form, the lowest grade within a group determines the group grade and the worst of the first three group grades determines the final evaluation. Arthrometric measurement (KT2000) was only done post operatively due to availability of the apparatus. The comparison with the normal knee (since all patients had normal contralateral knee) is performed and documented. We obtained arthrometric measurement in 7 of 9 patients (one patient could not come because of distance constraint and another patient refused assessment). Arthrometric evaluation was done using KT-2000 at maximum force of 30 pounds to both knees at the angle of $90^{\circ}$.

\section{RESULTS}

\section{Dermographic Distribution}

The age of patients ranged from 18 to 36 years. The mean age was 26 . One patient was below 20 years old and 3 were above 30 years old. Eight patients (89\%) were male. This is most probably due to more active lifestyle and involved with high energy sports. Seven patients were Malay and two patients were Chinese.

\section{Etiology}

Six patients sustained the injury during road traffic accident; the remaining patients sustained injury during sport at National level (two hockey players and one athlete in long jump).

\section{Associated Injuries}

Eight patients had associated injury including four lateral collateral ligament and posterior lateral complex, 2 medial collateral ligament, 1 anterior cruciate ligament partial tear and 1 medial meniscus tear. Posterior lateral instability was reconstructed using Clancy $^{5}$ technique. Medial collateral ligament injuries were treated conservatively. Medial meniscus tear was repaired with outside-in technique.

\section{Side of Injury}

The right knee was injured more than left. This correlated with dominant use of right leg. All three patients from sport injury group had injury to the dominant limbs (2 right side and 1 left side), whereas, in road traffic accident group four out of six patients injured the dominant limbs (4 right side and 2 left side).

\section{Timing of Surgery}

The timing of surgery ranged from 3 months to 40 months, with a mean of 17.2 months. All injuries were chronic and had undergone physiotherapy prior to the decision for surgery was. Clancy ${ }^{8}$ found that timing of surgery did not affect the outcome of surgery. However, we observed that eight of our cases had osteoarthritis of the knee at the time of surgery; two patients with Grade 1 and six patients with Grade 2 according to Outerbridge classification.

\section{Assessment Results}

Assessment was performed for subjective results using Lysholm Knee Score and IKDC knee examination score; and for objective results using arthrometric measurement KT-2000.

\section{Lysholm Knee Score}

The score $95-100 \%$ is consider Excellent, $84-94 \%$ is Good, $65-83 \%$ is Fair and less than $64 \%$ is Poor. At 12 months, 8 patients (89\%) had fair grade and 1 patient (11\%) had poor grade. Postoperatively at 24 months, 1 patient (11\%) had excellent and 4 patients (44\%) had good result. The patients who obtained poor result at 12 months were continued to have poor result at 24 months. The best result obtained from patient with isolated posterior cruciate ligament tear. Overall 
patients with excellent and good grades were $55 \%$. The poor result at 12 months mainly because of pain and slow rehabilitation.

\section{IKDC knee examination score}

The worst score in the knee examination including motion, laxity and pain determines the final grade evaluation. At 24 months, 1 patient (14.3\%) out of 7 was categorized grade $A$ and 4 patients $(59.1 \%$ ) grade B. Overall $73.4 \%$ patients had either grade A or B.

\section{Arthrometric Measurement}

KT-2000 arthrometric measurement was performed on 7 patients. The results showed that only one patient had similar measurement with the normal side. Three patients had $3 \mathrm{~mm}$ or less difference. Others had more than $3 \mathrm{~mm}$ but less than $6 \mathrm{~mm}$ differences. The entire patients tested has solid end point, which suggest that there is no evidence of graft dislodgement or rupture. Measurement result is not proportionate to the subjective assessment result.

\section{Complications}

There were two complications related to the surgical procedure; one patient had superficial infection and another had screw protrusion at the posterior tibial edge site. Four patients had pain over the donor site of the graft, two were mild and the other two were moderate at 12 months follow up. There was no failure due to graft rupture in our series. No other complications noted at 24 months follow up.

\section{DISCUSSION}

Injuries of the posterior cruciate ligament are not as common as anterior cruciate ligament but more frequent than previous estimates. The outcome of the posterior cruciate ligament reconstruction is also not as good as anterior cruciate ligament reconstruction. ${ }^{9}$ The results of posterior cruciate ligament reconstruction are inconsistent and far from good with conventional surgical technique. Lately, more studies have been published concerning basic science and newer techniques of posterior cruciate ligament reconstruction. These include introduction of the double bundle technique and tibial inlay technique.

The aim of this study is to evaluate the result of posterior cruciate ligament reconstruction done in 9 young patients at a single institution by using single bundle technique. All cases were done with arthroscopic assistance, using ipsilateral bonepatella tendon bone graft. All the patients had pain and instability with Grade 3 laxity preoperatively. Radiographic degenerative changes were mostly seen in the patellofemoral compartment. However, osteoarthritis changes were not visualized in the plain films.
Our results are moderate, with subjective satisfaction of $55 \%$ and clinical satisfaction and objective measurement of $73.4 \%$. These results are comparable with previous studies. ${ }^{4}$ However, our objective results are better than the subjective clinical satisfaction. There have been numerous operative procedures described, each with their own proponents, but in general theresults of all the operations are still inconsistent. There may be reasons for these poor results, including poor surgical technique, inaccurate tunnel placement, and proper graft choice. Although a new trend is toward double bundle posterior cruciate ligament reconstruction, the debate between single bundle and double bundle posterior cruciate ligament reconstruction will continue until sufficient data with longer follow-up become available to support predominance of one over the other. A study by $\mathrm{Li}$ revealed that although both techniques resulted in similar patient satisfaction as measured by outcome assessment, the double bundle procedure significantly improved knee stability. ${ }^{10}$ Recently, remnant preservation PCL reconstruction technique has been introduced and a systematic review showed that it produced satisfactory outcome. ${ }^{11}$

The indications for surgery in our patients are mainly pain and instability, and most of these patients were unable to perform daily activities because of the symptoms. Prolonged instability with combine ligamentous injuries and development of degenerative osteoarthritis are the most possible causes of these patients' symptoms. Clinical evidence exists that most non-operative treated posterior cruciate ligament injuries heal, provided that proper protocol is followed, the knee is kept in a brace with the tibial in reduced position and good patient compliance. ${ }^{12}$ Most of our patient came late or were referred by other institutions after having tried conservative treatment for a period of time. In addition, there was no proper non-operative treatment protocol.

Many authors reported that results of surgical reconstruction for isolated grade 3 posterior cruciate ligaments are generally better than that of combined posterior cruciate ligament and posterolateral reconstructions of the knee. ${ }^{4}$ The combined posterior cruciate ligament and posterolateral instabilities frequently result in functional disability, deterioration of the articular cartilage and degenerative changes of the affected knees. Successful posterior cruciate ligament surgery depends upon recognition and treatment of posterolateral complex injuries. Four of the patients in this series sustained combined injuries to the posterior cruciate ligament and posterolateral complex or lateral collateral ligament. These patients were operated relatively earlier with the mean of 8.7 months compared to overall, which is 17.3 months. Out of four, one patient obtained good and three patients had fair result. 
The graft used in our study was bone-patella tendonbone autograft, taken from ipsilateral knee. The advantages of using this graft include is that it is readily available, better graft healing of both femoral and tibial side due to bone to bone contact allowing for earlier rehabilitation. However, the difficulty is the passage of the bone block around the back of the tibia and a relatively smaller graft that is weaker compared to posterior cruciate ligament itself. There is no difference in outcome between autograft and allograft for posterior cruciate ligament reconstruction. ${ }^{13}$ Besides allograft, synthetic allograft also can be used for PCL reconstruction with comparable result. ${ }^{14}$ However, four of our patients experienced donor site pain especially on kneeling even after 1 year of follow up. A study by Lin et al also gives similar result when comparing patellar tendon graft and hamstring tendon graft in PCL reconstruction. ${ }^{15}$ The choice of graft is mostly based on surgeon preference and depends on the availability of the graft.

The limitation of this study is that it is retrospective of a small number of patients with short term follow up. There were no uniform protocols for preoperative physiotherapy since the cases are referred late to our centre. The technique needs to be improved either using more biomechanical sound technique or anatomically position of tunnel. A prospective study would be good to further evaluate the efficacy of the procedure.

\section{CONCLUSION}

This surgical technique only has moderate success in restoration of ligament stability with $73.4 \%$ satisfactory objective results and good clinical assessment postoperatively. However, the functional improvement and patient satisfaction is only $55 \%$. There appears to be poor correlation between patient satisfaction and objective measurement.

\section{REFERENCES}

1. Shahrulazua A, Rafedon M, Mohd Nizlan MN, Sullivan JA. Delayed compartment syndrome of leg and foot due to rupture of popliteal artery pseudoaneurysm following posterior cruciate ligament reconstruction. BMJ Case Rep;2014.

2. Shelbourne KD, Jennings RW, Vahey TN. Magnetic resonance imaging of posterior cruciate ligament injuries: assessment of healing. Am J Knee Surg 1999;12:209-13.

3. Gollehon DL, Torzilli PA, Warren RF. The role of the posterolateral and cruciate ligaments in the stability of the human knee. A biomechanical study. J Bone Joint Surg Am 1987;69:233-42.

4. Wang CJ, Chen HS, Huang TW. Outcome of arthroscopic single bundle reconstruction for complete posterior cruciate ligament tear. Injury 2003;34:747-51.

5. WG C. Repair and recosntruction of the posterior cruciate ligament. In: MW C, ed. Operative
Orthopaedics. Philadelphia: JB Lippincott; 1988:1651-65.

6. (Accessed oct 13, 2015, at http://www.lonnie paulosmd.com/pdf/Group_B_-_KNEE_PCL_ Protocol.pdf.)

7. Tegner Y, Lysholm J, Odensten M, Gillquist J. Evaluation of cruciate ligament injuries. A review. Acta Orthop Scand 1988;59:336-41.

8. Clancy WG, Jr., Shelbourne KD, Zoellner GB, Keene JS, Reider B, Rosenberg TD. Treatment of knee joint instability secondary to rupture of the posterior cruciate ligament. Report of a new procedure. J Bone Joint Surg Am 1983;65:310-22.

9. Cross MJ, Powell JF. Long-term followup of posterior cruciate ligament rupture: a study of 116 cases. Am J Sports Med 1984;12:292-7.

10. Li Y, Li J, Wang J, Gao S, Zhang Y. Comparison of single-bundle and double-bundle isolated posterior cruciate ligament reconstruction with allograft: a prospective, randomized study. Arthroscopy;30:695-700.

11. Song JG, Kim HJ, Han JH, et al. Clinical Outcome of Posterior Cruciate Ligament Reconstruction With and Without Remnant Preservation. Arthroscopy.

12. Shelbourne KD, Gray T. Natural history of acute posterior cruciate ligament tears. J Knee Surg 2002;15:103-7.

13. Fanelli GC, Giannotti BF, Edson CJ. The posterior cruciate ligament arthroscopic evaluation and treatment. Arthroscopy 1994;10: 673-88.

14. Xu X, Huang T, Liu Z, et al. Hamstring tendon autograft versus LARS artificial ligament for arthroscopic posterior cruciate ligament reconstruction in a long-term follow-up. Arch Orthop Trauma Surg 2014 Dec;134(12):1753-9 doi: 101007/s00402-014-2104-7 Epub 2014 Nov 7.

15. Lin YC, Chen SK, Liu TH, Cheng YM, Chou PP. Arthroscopic transtibial single-bundle posterior cruciate ligament reconstruction using patellar tendon graft compared with hamstring tendon graft. Arch Orthop Trauma Surg;133:523-30. 
